\title{
An Ant Colony based Routing Algorithm for Wireless Sensor Network
}

\author{
Xiaodong Liu, Songyang Li, Miao Wang \\ School of Computer, Henan Institute of Engineering, Zhengzhou, 451191, China \\ E-mail: liuxiaodongxht@qq.com,lsycqu@cqu.edu.cn,wmscan@tom.com
}

\begin{abstract}
Wireless sensor network (WSN) is a new research area of computer science and technology and has a wide application future, more and more attention. It will be much market in application of military, health, home, environment, etc. In WSN, sensor devices have limited supply of energy. The sensor death due to dissipating battery energy is one of the fundamental design issues in WSNs. Hence, energy efficiency is argued to be the most important requirement for WSN. Aiming at the limitation of energy of wireless sensor network node, this paper presents a routing algorithm which is based on the ant's capability of finding the best paths between a food source and their nests. The pheromone and the energy of the node and delay of the node were combined to affect the pheromone concentration in optimization. Simulation results show that it can balance energy consumption between nodes and prolong the lifecycle of the whole network.
\end{abstract}

Keywords: routing algorithm, wireless sensor network, ant colony

\section{Introduction}

WSNs consist of a large number of inexpensive devices that are networked via wireless communication subsystems, which is used to collect and send various kinds of messages to a base station [1-2]. It is made by the convergence of sensor, nested computation, networks and wireless communication technologies [3], and it is a new research area of computer science and technology and has a wide application future [4]. A WSN differs from traditional network in four principal aspects. First, the number of nodes is very large and there exists a lot of redundancy data; second, node energy is difficult to recharge; third, network topology is easy to change; and four, there is no strict control center in the network. The energy efficient clustering and routing are the challenging research issues in WSN due to low battery life, computational overhead, self organization and limited transmission range of sensor nodes.

How to efficiently use of sensor battery capacity to increase the life time of the network has been a research focus [5-6]. Several clustering and routing algorithms are reported so far. In [7], dynamic layered Voronoi scoping and dynamic anchor selection are integrated to effectively reduce the dissemination scopes and frequencies of routing updates as the sinks move in the network. Rachamalla, S et $a l$. [8] developed a framework for power-control and delay-aware routing and MAC protocol for Energy constraint and delay sensitive wireless sensor network applications, which aims to route the packets along multiple paths with the delay. In [9] the BS maintains a list of $\mathrm{CHs}$ based on the energy. The proposed algorithm decreases the number of packet reception between $\mathrm{CHs}$ and cluster members to minimize the energy consumption but not assured to form disjoint clustering which increases the possibility of data loss. Recently, the routing protocols of WSN begin to develop in the direction of intelligence. Barolli et al. proposed an intelligent fuzzy-based cluster head selection algorithm in [10]. It uses three input parameters 
for fuzzy logic control during $\mathrm{CH}$ selection. An energy based ant colony optimization algorithm for WSN is proposed in [11-12]. But the adaptive behavior of the scheduling problem after the addition of new tasks to the original schedule and after the mobility of sensor node or the router is not addressed.

The recent work mainly considers energy consumption. However, performance indicators such as network delay, congestion, transmission efficiency, robustness, etc are also need to be considered. In this paper, we propose a routing protocol for WSM which is based on improved ant colony system. It can balance the energy consumption of the sensor nodes and prolong the lifecycle of the whole network. It can also effectively alleviate the network congestion and reduce the average delay.

The remainder of this paper is organized as follows: The system model is described in section 2. Section 3 introduces our routing algorithm based on ant colony system. Section 4 shows the experimental results. Finally, section 5 summarizes our conclusions and suggests future work.

\section{System Model}

\subsection{Energy Consumption Model}

The energy consumption model may affect the advantages and performance of the protocol. In order to facilitate comparison, we use the energy consumption model which is used in the LEACH routing protocol [13]. Fig.1 shows the energy consumption model.

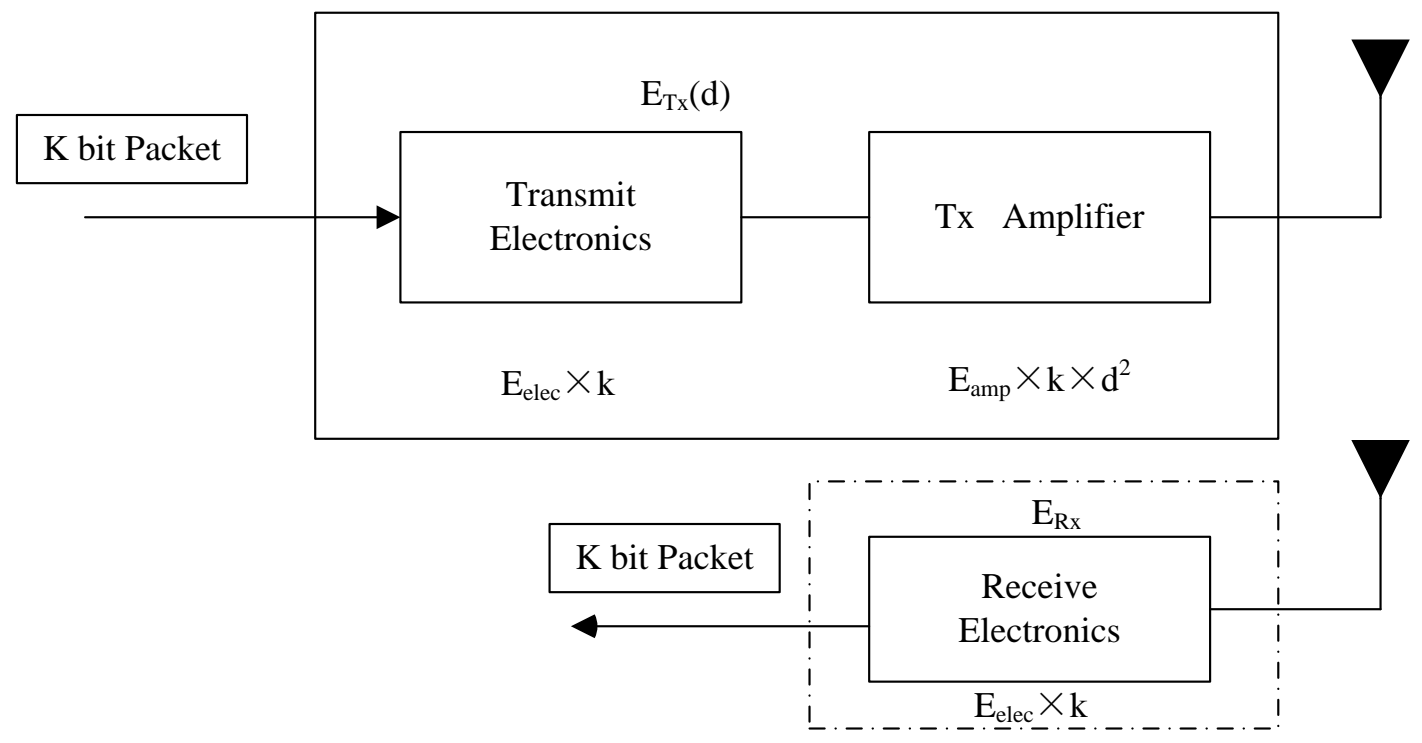

Figure 1. Energy Consumption Model

The energy consumption of transmission and acceptance $E_{\text {elec }}=50 \mathrm{~nJ} / \mathrm{bit}$. Unit energy consumption of transmission amplification $E_{a m p}=100 \mathrm{pJ}\left(\mathrm{bit} \times \mathrm{m}^{2}\right)$. Unit energy consumption of data fusion $E_{\text {fuse }}=5 \mathrm{~nJ} / \mathrm{bit}$. The energy consumption of transmitting data depends on the data size and the transmission distance. The energy consumption of receiving data only depend on the size of the data. Thus, when a node send $\mathrm{k}$ bit data to another node when distance between them is $\mathrm{d}$, the energy consumption is

$$
E_{T x}(k, d)=E_{\text {elec }} \times k+E_{\text {amp }} \times k \times d^{2}
$$

The energy consumption of receiving is

$$
E_{R x}(k)=E_{\text {elec }} \times k
$$


The energy consumption of data fusion is

$$
E_{\text {fuse }}(k)=E_{\text {fuse }} \times k
$$

\subsection{Network Model}

In order to make our routing algorithm more universal, our network model assume that the sensor nodes are randomly distributed in a rectangular area. At the same time, we make following assumptions for sensor nodes:

(1) The initial energy of all nodes is the same.

(2) Each node can obtain the information of its neighbor nodes.

(3) The number of nodes in the network is very large.

\subsection{The Model of Ant Colony Algorithm}

Ant colony algorithm was first proposed in the solution of the TSP problem. Given a TSP problem with $\mathrm{n}$ city and the number of ants in the ant colony is $\mathrm{m}$. In order to simulate the behavior of real ants, we first introduce the following notations:

(1) $m$ is the number of ant in the ant colony.

(2) $b_{i}(t)$ is the number of ant of city $\mathrm{i}$ at the moment of $\mathrm{t}$.

(3) $d_{i j}$ is the distance between city $\mathrm{i}$ and $\mathrm{j}$.

(4) $\eta_{i j}$ is the visibility of edge $(i, j)$, which reflect the degree of inspiration that a node transferred from city $\mathrm{i}$ to $\mathrm{j}$. Generally, $\eta_{i j}$ is $\eta_{i j}=\frac{1}{d_{i j}}$.

(5) $\tau_{i j}$ is the pheromone intensity of edge $(\mathrm{i}, \mathrm{j})$.

(6) $\alpha$ is the relative importance degree of pheromone.

(7) $\beta$ is the relative importance degree of visibility.

(8) $p_{i j}^{k}$ is the transfer probability of ant $\mathrm{k}$, where $\mathrm{j}$ is the city not yet visited.

Secondly, the behavior of each ant should conform to the following rules:

(1) Ant select the next path according to the pheromone concentration on the path.

(2) The path which has gone cannot be selected. It is controlled by a data table list.

(3) Once a cycle has been completed, pheromone concentration of the whole path is released, and pheromone concentration on the path is updated.

In the initial stage, all the edges are given a certain pheromone. Ant $\mathrm{k}(\mathrm{k}=1,2, \ldots \mathrm{m})$ determine the direction according to the amount of pheromone on each path. Tabu list $\operatorname{tabu}_{k}(\mathrm{k}=1,2, \ldots \mathrm{m})$ is used to record nodes which ant $\mathrm{k}$ has been traveled. In the search process, state transition probability is calculated according to the pheromone and heuristic information of each path. At the time t, transfer probability $p_{i j}^{k}(t)$ can be calculated by the following formula (4).

$$
p_{i j}^{k}= \begin{cases}\frac{\tau_{i j}^{\alpha}(t) \eta_{i j}^{\beta}(t)}{\sum_{s \text { eallowed }_{k} \tau_{i s}^{\alpha}(t) \eta_{i s}^{\beta}(t)}}, j \in \text { allowed }_{k} \\ 0 \quad, \text { otherwise }\end{cases}
$$

where $\tau_{i j}(t)$ is the amount of pheromone, allowed $_{k}=\left\{C-\right.$ tabu $\left._{k}\right\}$ is nodes that can be selected in the next.

In order to avoid there is too much residual pheromone which flood inspired information, residual pheromone will be updated after completing the traversal of all nodes. The pheromone will be updated according to the following formula.

$$
\tau_{i j}(t+n)=(1-\rho) \tau_{i j}(t)+\Delta \tau_{i j}(t)
$$




$$
\Delta \tau_{i j}(t)=\sum_{k=1}^{m} \Delta \tau_{i j}^{k}
$$

where $\rho$ is evaporation coefficient of pheromone, $1-\rho$ is residual coefficient of pheromone, $\Delta \tau_{i j}(t)$ is increased pheromone of edge $(\mathrm{i}, \mathrm{j})$

$$
\mathrm{V} \tau_{i j}^{k}= \begin{cases}\frac{Q}{L_{k}} & , \text { if ant k select edge }(\mathrm{i}, \mathrm{j}) \text { in the } \mathrm{k} \text { cycle } \\ 0 & , \text { else }\end{cases}
$$

where $\mathrm{Q}$ is a constant, its value is the total amount of pheromone, $L_{k}$ is the length of path of ant $\mathrm{k}$ in this cycle.

\section{Routing Algorithm based on Improved Ant Colony System}

\subsection{Improvement of Ant Colony Algorithm}

From the above ant algorithm, we can see that ant reach the destination by means of the most pheromone path. If a path is the optimal path to the destination, the number of ant in the path will be huge. This will lead to the node energy of the network on the path decrease sharply and shorten the life cycle of the whole network. In order to solve these problem, ant colony algorithm muse be improved to make the energy consumption of the nodes is relatively balanced.

This paper is improved from the following three aspects.

(1) $p_{i j}^{k}$ is determined by the following formula

$$
p_{i j}^{k}= \begin{cases}\frac{\tau_{i j}^{\alpha} \eta_{i j}^{\beta} E_{j}}{\sum_{\text {seallowed }_{k}} \tau_{i s}^{\alpha} \eta_{i s}^{\beta} E_{s}} & , j \in \text { allowed }_{k} \\ 0 & , \text { otherwise }\end{cases}
$$

where $\tau_{i j}(t)$ is pheromone intensity, $\eta_{i j}(t)$ is the inverse of the distance from the node $\mathrm{i}$ to node $\mathrm{j}, E_{j}$ is the residual energy of node that ant $\mathrm{k}$ will visit, allowed $_{k}$ is the set that ant $\mathrm{k}$ has not visit.

Energy control factor is added in the formula (8). The energy consumption of nodes is balanced and the lifecycle of the whole network is prolonged. The delay factor is also considered in the formula (8). The delay can effectively reflect the congestion level of the network. Thus, our algorithm can effectively alleviate network congestion.

(2) Aiming at the network topological structure is variable, ant $\mathrm{k}$ which locates in the node i select the next node according to the following formula

$$
p_{i j}^{k}=\left\{\begin{array}{lc}
1 / \mathrm{Ln}, & \text { if } \mathrm{q} \leq \mathrm{q} 0 \\
\text { calculate } p_{i j}^{k} & \text { by formula }(8), \text { else }
\end{array}\right.
$$

where $\mathrm{q}$ is a random number and $q \in(0,1), L_{n}$ is the number of neighbors of node $\mathrm{n}$. We can decide whether to search the new path according to the pheromone or explore the new path by set the value of $q_{0}$.

As can be seen from the formula (9), if the value of $q_{0}$ is large, ants are easy to choose edges which pheromone is large. This can lead to the algorithm is easy to fall into local optimal solution. If the value of $q_{0}$ is small, the search space can be expanded. But the search has a certain blindness and the algorithm is not easy to converge. Comprehensive consideration of these two aspects, the value of $q_{0}$ is dynamic adjustment in the iterative process. $q_{0}$ is determined by 


$$
q 0=\left\{\begin{array}{l}
c, 0<\text { cycle } \leq \mathrm{n} 0 \\
c 0, n 0<\text { cycle } \leq \mathrm{n} 1 \\
\mathrm{c}, \mathrm{n}_{1<\mathrm{Cycl}} \mathrm{e} \leq \mathrm{n}_{\max }
\end{array}\right.
$$

where $n_{0}, n_{1}$ are the number of iterative, $n_{\max }$ is the maximum number of iterative, $0.7<c<0.9,0<c 0<0.3$. When the algorithm starts running, edges which pheromone is large are easy to be selected. After iterative $n_{0}$, in order to avoid falling into local optimal solution, the value of $q_{0}$ is changed to expand search space. After iterative $n_{1}$, in order to make the algorithm converge to the global optimal solution, the value of $q_{0}$ is changed again.

(3) Loop is also need to be avoided in ant colony algorithm. In this paper, hop count information is recorded at the expense of a very small space. In the route preparation stage, nodes around the Sink node (suppose K) first to know that sink node in their own adjacency node table. The number count of these nodes is set to 1 . At the same time, these nodes will send their own jump number information to their adjacency nodes. When adjacency nodes $\mathrm{K}+1$ receive the information, they will set their jump number and send their own jump number information to their adjacency nodes.

When a node selects the next node, the number of jump number must larger than itself. It can speed of convergence and avoid loop.

\subsection{The Design of Route Algorithm}

Step 1. Route preparation stage. Sink node broadcast an initialization signaling. Each node will get its own adjacent node and add it to its own route table. The value of pheromone of adjacent node link set to 1 . Each node generates the current node's ant pack which includes number of node and route table. The maximum number of iterations is set to $N_{\max }$, and the initial iteration number is set to 1 .

Step 2. Route establishment stage. The Ant which locates in node i will select the next node according to formula (8)-(10). When a node receive the ant package, it will forward ant package according the probability $p_{i j}^{k}$.

Step 3. Route optimization stage. Once sink node receives ant package, it will count how many ants pack sent by each node. Suppose the total number of node is $\mathrm{m}$ and the number of ants package sent by node $i$ is $X_{i}(i=1, \ldots, m)$.

The total amount of ant package of network can be expressed by.

$$
T=\sum_{i=1}^{m} X_{i}
$$

The mathematical expectation of the amount of ant packet transmission is

$$
E(x)=\sum_{i=1}^{m} \frac{X_{i}}{T} X_{i}
$$

The variance of the amount of the ant packet is

$$
D(x)=\sum_{i=1}^{m}\left(X_{i}-E X\right)^{2} \frac{X_{i}}{T}
$$

The Evaluation function of the amount of the ant packet can be defined by the following formula

$$
F(x)=E(x) D(x)^{\tau}
$$

where $\tau$ is a adjustable parameters. The weight of $\mathrm{E}(\mathrm{x})$ and $\mathrm{D}(\mathrm{x})$ can be adjusted by $\tau$.

Each node generates back ant package. When nodes receive back ant package, adjacency link information of back ant package will be updated by

$$
\tau_{i j}(t+n)=(1-\rho) \tau_{i j}(t)+\Delta \tau_{i j}(t)
$$




$$
\Delta \tau_{i j}(t)=\frac{F(x)-F \min (x)}{F(x)+F \min (x)}
$$

The node which locates in the back ant package is selected as the next hop. At the same time, whether to send a new packet of ants is determined according to the information in the back ant packets. Route is established when new ant package need not send.

\section{Performance Evaluation}

In order to evaluate the performance of our proposed algorithm, we compare the performance of our ant colony based routing algorithm (ACRA) with LEACH algorithm. All of our experiments are tested on an quad-core Intel Core i5 2.6 GHz with $8 \mathrm{G}$ RAM under a Microsoft Windows 8 environment and all the experiments were implemented in MATLAB R2014b. We select 50 randomly distributed nodes in the range of $200 \times 200$. Suppose the data transmitted and received by each node is 4000 bit. Base station is located in $(100,200)$. Initial energy of each node is 0.5 .

In order to study the influence of parameters $\alpha, \beta, \rho$ on the performance of our algorithm, we first evaluate the performance of our algorithm when the value of $\alpha, \beta, \rho$ variable. Each experiment was conducted 50 times and take the average value in order to avoid accidental.

In the first set of experiment, we fix the range of experiment is $200 \times 200$. The number of nodes is 50. We fix the value $\beta=2$ and $\rho=0.5$. The value of $\alpha$ changed from 1 to 10 . The life cycle of the first node is recorded. Repeat the experiment 50 times and take the mean value. Fig. 2 shows the relationship between life cycle of the first node and the value of $\alpha$. The $\mathrm{x}$-axis is the value of $\alpha$ and the $\mathrm{y}$-axis is the life cycle of the first node.

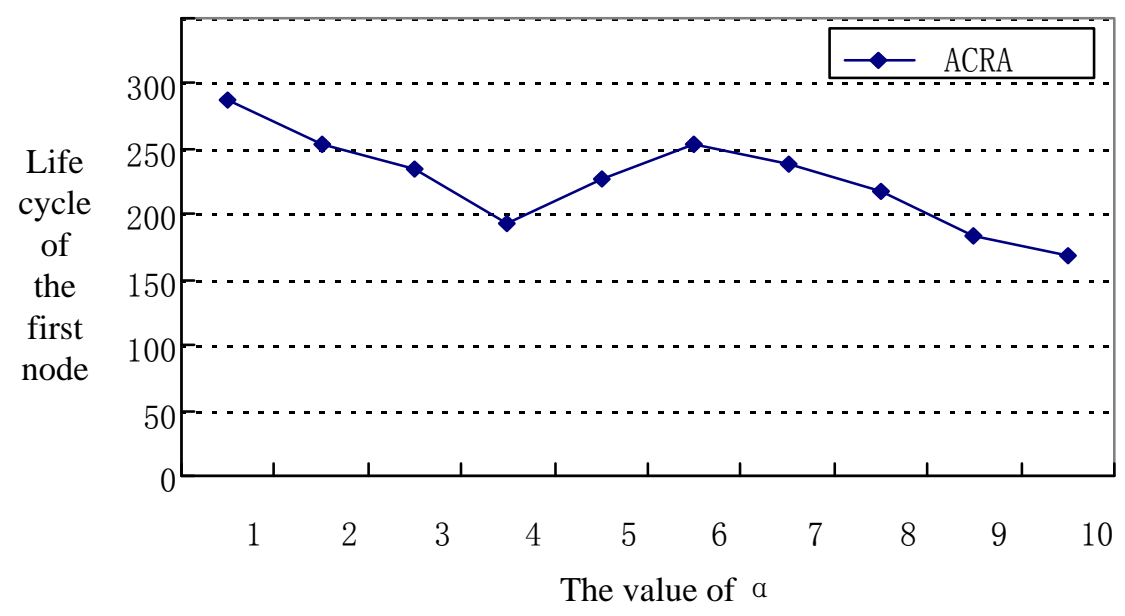

\section{Figure 2. The Relationship between Life Cycle of the First Node and the value of $\alpha$}

As we can see from the Fig.2, when the value of $\alpha$ is lower, the cycle of life of the first node is longer. There are two main reasons for this. one is because the proportion of the energy factor is relatively large. The other is because the randomness of route choice is relatively large when the value of $\alpha$ is lower. If the value of $\alpha$ too big, convergence can be accelerated, but randomness of search is weaken. Thus, the value of $\alpha$ cannot too big or small. 
In the second set of experiment, we fix the range is $200 \times 200$. The number of nodes is 50 . We fix the value $\alpha=1$ and $\rho=0.5$. The value of $\beta$ changed from 1 to 8. The life cycle of the first node is recorded. Repeat the experiment 50 times and take the mean value. Fig. 3 shows the relationship between life cycle of the first node and the value of $\beta$. The $\mathrm{x}$-axis is the value of $\beta$ and the $\mathrm{y}$-axis is the life cycle of the first node.

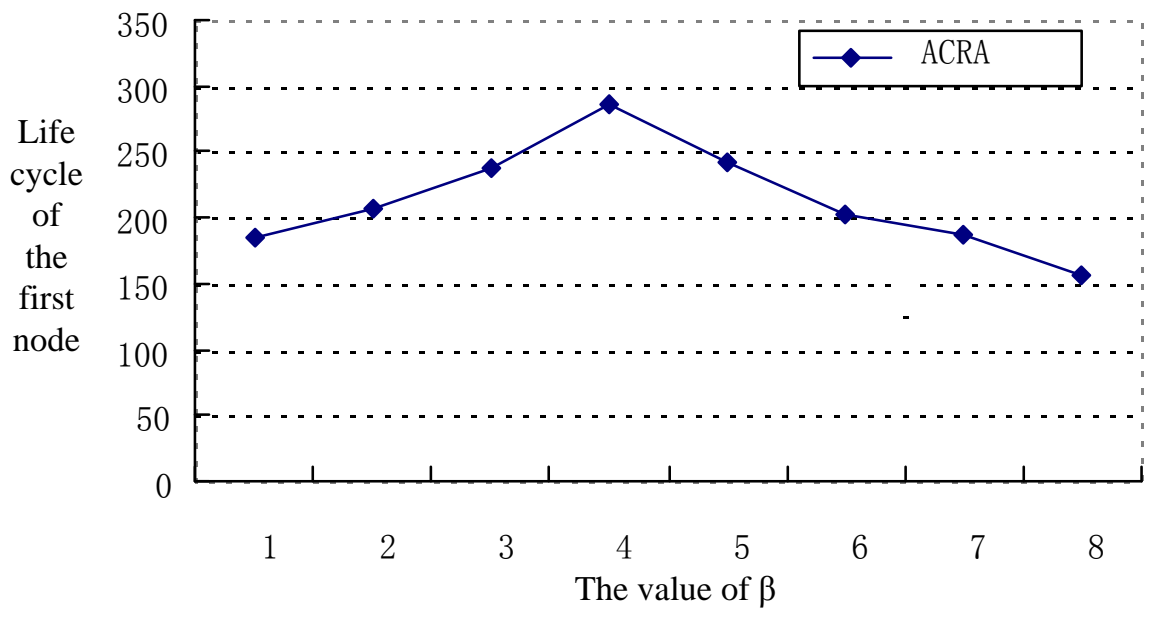

Figure 3. The Relationship between Life Cycle of the First Node and the Value of $\beta$

As we can see from the Fig.3, heuristic factor $\beta$ reflects the relative importance of heuristic information in guiding the process of ant colony search. The value of $\beta$ reflects the relative importance of apriority and certainty. When the value of $\beta$ is small, the randomness of selection of ant colony is relatively large. Thus, the optimal path cannot be easily found and the life cycle of the first node is short. When the value of $\beta$ is big, convergence performance of algorithm is relatively bad.

In the third set of experiment, we fix the range of experiment is $200 \times 200$. The number of nodes is 50. We fix the value $\alpha=1$ and $\beta=2$. The value of $\rho$ changed from 0.1 to 0.9 . The life cycle of the first node is recorded. Repeat the experiment 50 times and take the mean value. Fig. 4 shows the relationship between life cycle of the first node and the value of $\rho$. The $\mathrm{x}$-axis is the value of $\rho$ and the $\mathrm{y}$-axis is the life cycle of the first node. 


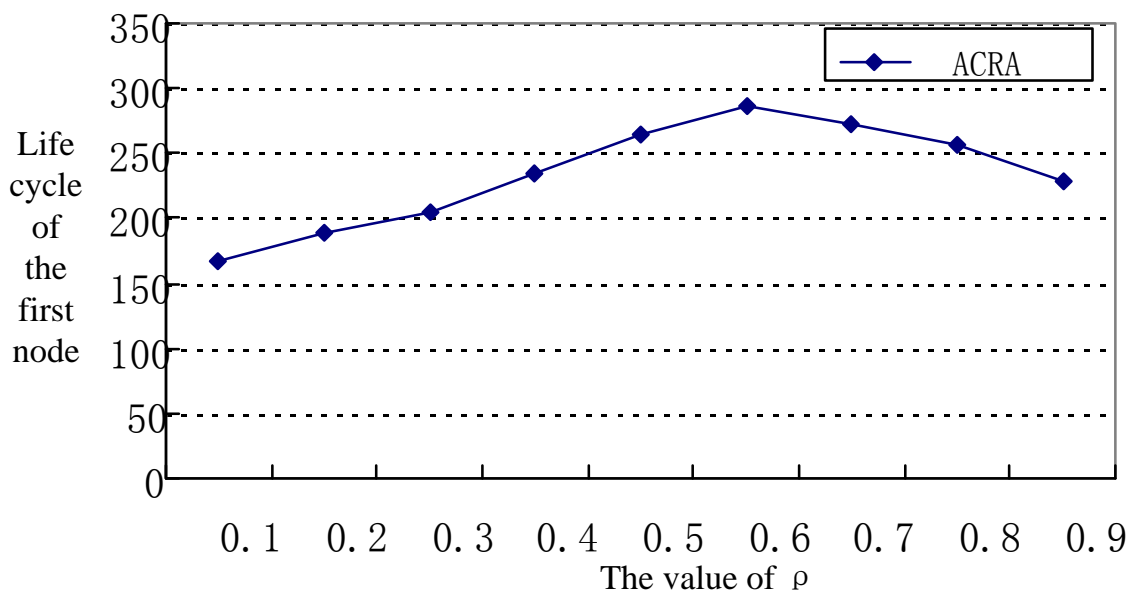

Figure 4. The Relationship between Life Cycle of the First Node and the Value of $\rho$

As we can see from the Fig.4, when the value of $\rho$ is small, pheromone volatile slow and incorrect pheromone cannot be fast volatile. This will lead to ant colony cannot find new better solutions and the life cycle of the first node is short. When the value of $\rho$ is big, incorrect pheromone volatile fast and ant colony can find better solutions more easily. The life cycle of nodes can be prolonged. But when $\rho$ increases to a certain value, legacy pheromone cannot be fully utilized. It will lead to the optimal solution is hard to find and the life cycle of nodes is short.

On the basis of simulation analysis of various parameters, we set the value of $\alpha=3, \beta=4$ and $\rho=0.7$. These parameters are based on our repeated tests. We compare the life cycle of nodes between ACRA and LEACH algorithm. We fix the range of experiment is $200 \times 200$ and the initial energy $Q=0.5$. The life cycle of the 1 th, 5th, 10th, 15th, 20th, 25th, 30th, 35th, 40th, 45th and 50th node is recorded (as is shown in Fig.5).

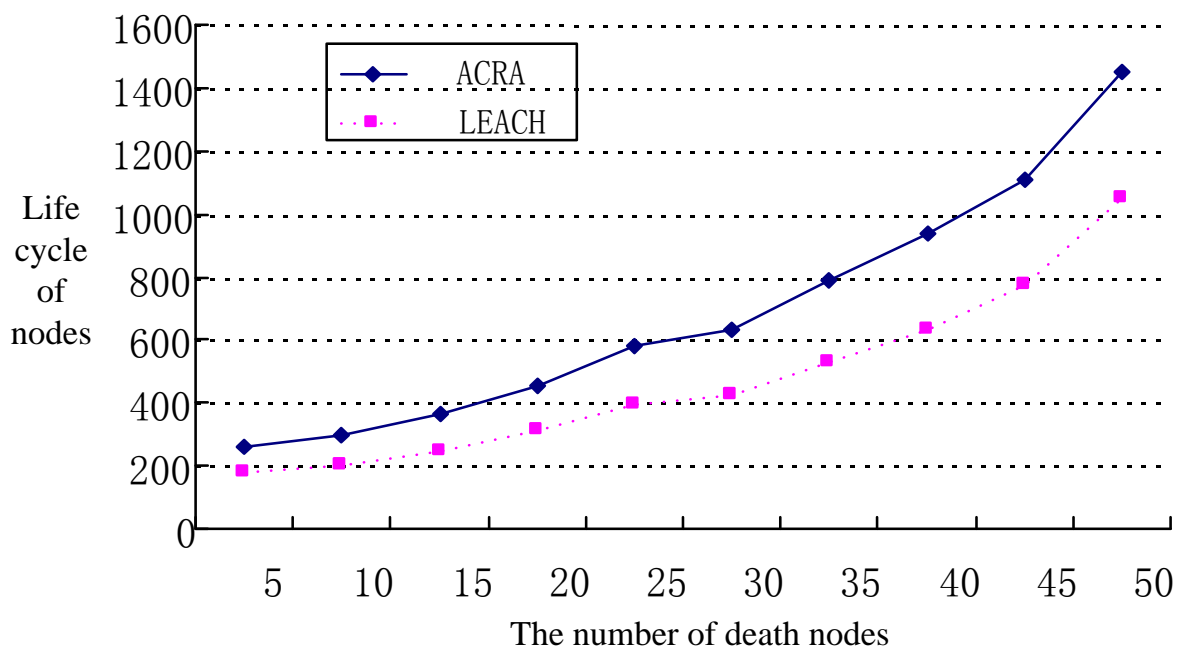

Figure 5. The Life Cycle of Nodes

Fig. 5 shows the life cycle of the 1th, 5th, 10th, 15th, 20th, 25th, 30th, 35th, 40th, 45th and 50th node under ACRA and LEACH. As we can see from the Fig.4, the life cycle of the improved ant colony algorithm is better than LEACH in the first 45 
nodes. Compared with ACRA, the energy consumption of LEACH is not balanced. This may lead to the residual energy of a small number of nodes is more, and their life cycle is long. As the remaining nodes are less, there is not much significance in the practical application.

As we can see from the Fig.5, our ACRA method makes the energy consumption of network relatively balanced and the life cycle of network is prolonged. There are two main reasons for this. One is because sensor nodes of ACRA can complete the route establishment themselves, which can save energy. The other is because some mathematical tools such as expectation and variance were introduced in our ACRA. This advantage will be more obvious with the increase of the size of the network. In order to verify this, we set run time of network $t=1200 \mathrm{~s}$, and set the range of network is $50 \times 50,100 \times 100,150 \times 150$ and $200 \times 200$ respectively. The LEACH algorithm and ACRA algorithm are respectively run under the same conditions (As is shown in Fig.6).

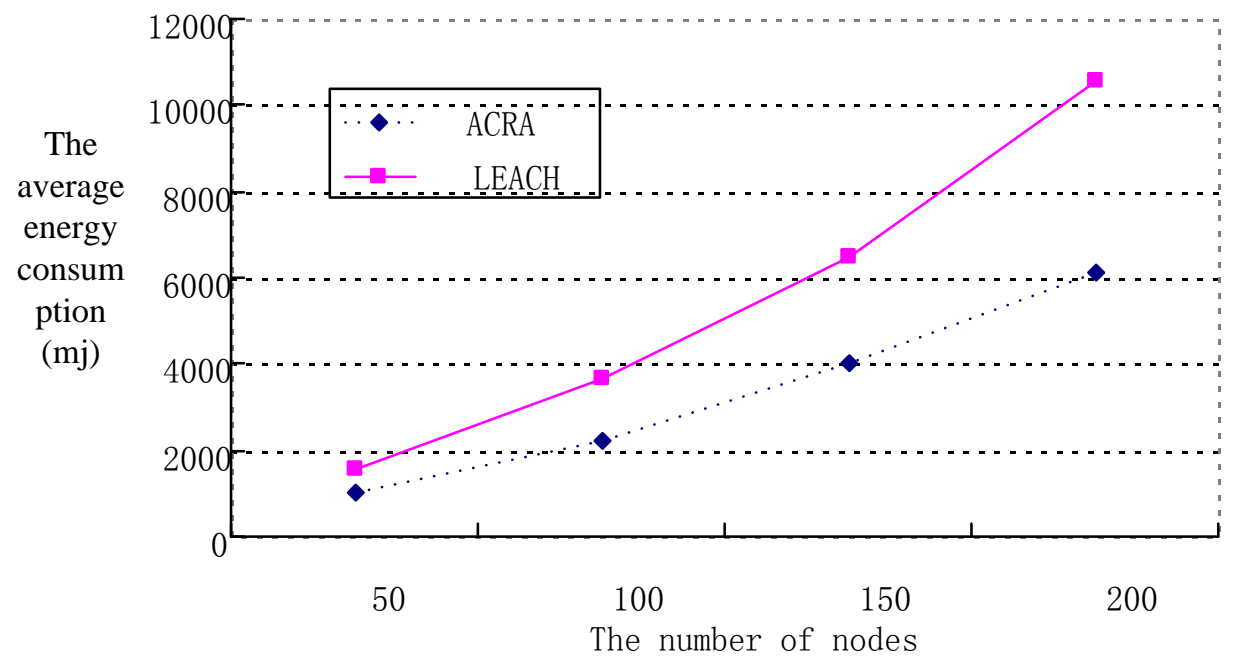

Figure 6. The Energy Consumption under Different Size of Network

In the LEACH algorithm, the energy consumption of the nodes is balanced by using the strategy of the first round of the cluster. The cluster head selection is carried out in the whole network, which waste lots of energy. In our ACRA, adaptive routing algorithm is adopted, which can use dynamic routing strategy according to the residual energy of nodes. It can protect the nodes which energy is close to exhaust and prolong the life cycle of network.

At the same time, in order to verify the convergence of the algorithm, we conducted 100 experiments under the same conditions. The results show that there are only 5 times without convergence to the optimal solution and fall into local optimal solution for the improved ant colony algorithm. This is because the hop selection mechanism and update method with the reward and punishment mechanism are introduced in our improved ant colony algorithm. Loop and local optimum problem of ant colony algorithm are avoided.

\section{Conclusion and Future Work}

Wireless Sensor network (WSN) which is made by the convergence of sensor, nested computation, networks and wireless communication technologies, is a novel technology about acquiring and processing information. It is a late-model wireless networks of infrastructure less network and can be used for testing, sensing, collecting and processing information of monitored objects. WSN is a new research 
area of computer science and technology and has a wide application future, more and more attention.

The energy efficient clustering and routing are the challenging research issues in WSN due to low battery life, computational overhead, no conventional, self organization and limited transmission range of sensor nodes. In this paper, we have proposed a routing algorithm which is based on the ant's capability of finding the best paths between a food source and their nests. The distinguished features of our algorithm compared to previous work are as follows: Firstly, the energy factor is added in the probability formula of ant colony algorithm, which can balance the energy consumption of nodes and prolong the life cycle of network. Secondly, aiming at the variable topology of WSN, parameters are dynamic adjusted during the search of path. Thirdly, pheromone update rules with reward and punishment mechanism are adopted, which can effectively avoid the problem that the ant colony algorithm is easy to be influenced by the early discovery of better solution. Simulation results show that it can balance energy consumption between nodes and prolong the lifecycle of the whole network.

There are also some shortcomings in our experiments, such as few nodes and network topology is relatively simple. In the future, we will consider large scale environment. In addition, the parameters are obtained by means of experiments. How to optimize these parameters and prove in theory is a topic worthy of our continuing research.

\section{Acknowledgements}

This paper is a revised and expanded version of a paper entitled [A Dynamic CPU Resources Scheduling Method for Xen Virtual Machine] presented at [The 4th International Conference on Next Generation Computer and Information Technology, October 22-24, 2014 at Qingdao, China]

This work is supported by Doctor Foundation of Henan Institute of Engineering (D2015022), Foundation of He'nan Educational Committee (15A520055), and a grant from National Science Fund for Young Scholars （No. 61301232)

\section{References}

[1] Z. Han, J. Wu and J. Zhang, "A General Self-Organized Tree-Based Energy-Balance Routing Protocol for Wireless Sensor Network”, IEEE Transactions on Nuclear Science, vol. 61, no. 2, (2014), pp. 732740.

[2] P. Wanga, H. Houa, X. He, "Survey on Application of Wireless Sensor Network in Smart grid", Procedia Computer Science, vol. 52, (2015), pp. 12-17.

[3] T. Miyazaki, P. Li and S. Guo, "On-demand Customizable Wireless Sensor Network". Procedia Computer Science, vol. 52, (2015), pp. 302-309.

[4] F. Jabeena and S. Nawaz, "In-network wireless sensor network query processors: State of the art, challenges and future directions", Information Fusion, vol. 25, (2015), pp. 1-15.

[5] S. Rani, J. Malhotra and R. Talwar, "Energy efficient chain based cooperative routing protocol for WSN", Applied Soft Computing, vol. 35, (2015), pp. 386-397.

[6] A. N. Z. Rashed, "High Transmission Bit Rate of Multi Giga Bit per second for Short Range Optical Wireless Access Communication Networks", International Journal of Advanced Science and Technology, vol. 32, (2011), pp. 23-32.

[7] L. Shi, Z. Yao and B. Zhang, "An efficient distributed routing protocol for wireless sensor networks with mobile sinks", International Journal of Communication Systems, vol. 28, no. 11, (2015), pp. 17891804.

[8] S. Rachamalla and A. S. Kancharla, "Power-Control Delay-aware routing and MAC protocol for Wireless Sensor Networks", 2015 IEEE 12th International Conference on Networking, Sensing and Control, (2015), pp. 527 - 532.

[9] Y. Guo, Y. Liu and Z. Zhang, "Study on the energy efficiency based on improved LEACH in wireless sensor networks", 2nd International Asia Conference on Informatics in Control, Automation and Robotics, (2010), pp. 388-390.

[10] L. Barolli, H. Ando and F. Xhafa, "Evaluation of an Intelligent Fuzzy-Based Cluster Head Selection System for WSNs Using Different Parameters", Proceedings of the 2011 IEEE Workshops of International Conference on Advanced Information Networking and Applications, (2011), 388-395. 
[11] T. C. Camilo, J. S. Carreto and F. Boavida, "An energy-efficient ant-based routing algorithm for wireless sensor networks", Ant Colony Optimization and Swarm Intelligence, Proceedings, (2006), pp. 49-59.

[12] W. Hongyuan, L. Zhiyuan and B. Ying, "Ant Colony Optimization Algorithm For WSN Cross-Layer Routing Protocol", Journal of Changzhou University, vol. 26, no. 2, (2014), pp. 32-37.

[13] S. Lindsey and C. S. Raghavendra, "PEGASIS: Power-efficient gathering in sensor information systems", The IEEE Aerosapce Conf.Montana:IEEE Aerosapce and Electronic Systems Society, (2002), pp. 1125-1130.

\section{Authors}

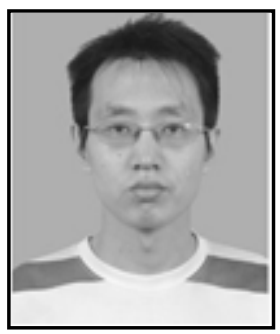

Xiaodong Liu, he received his Ph.D. degree from Shanghai University. He is currently an associate Professor of Henan Institute of Engineering. His primary research interests cover virtualization, cloud computing and grid computing.

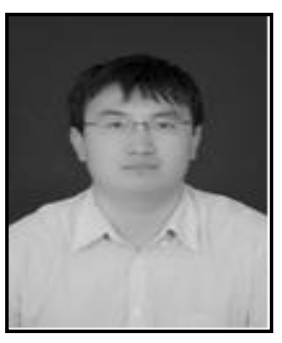

Songyang Li, he received his Ph.D. degree from Chongqin University. $\mathrm{He}$ is currently a lecturer of Henan Institute of Engineering. His primary research interests cover computer graphics, cloud computing

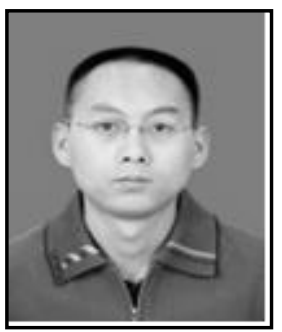

Miao Wang, he received the $\mathrm{PhD}$ degree in computer science from Harbin University of Science and Technology. Member of China Computer Federation. His research interests include Spatio-temporal database, Real-time database and Spatial Reasoning. 
International Journal of Future Generation Communication and Networking Vol. 9, No. 6 (2016) 